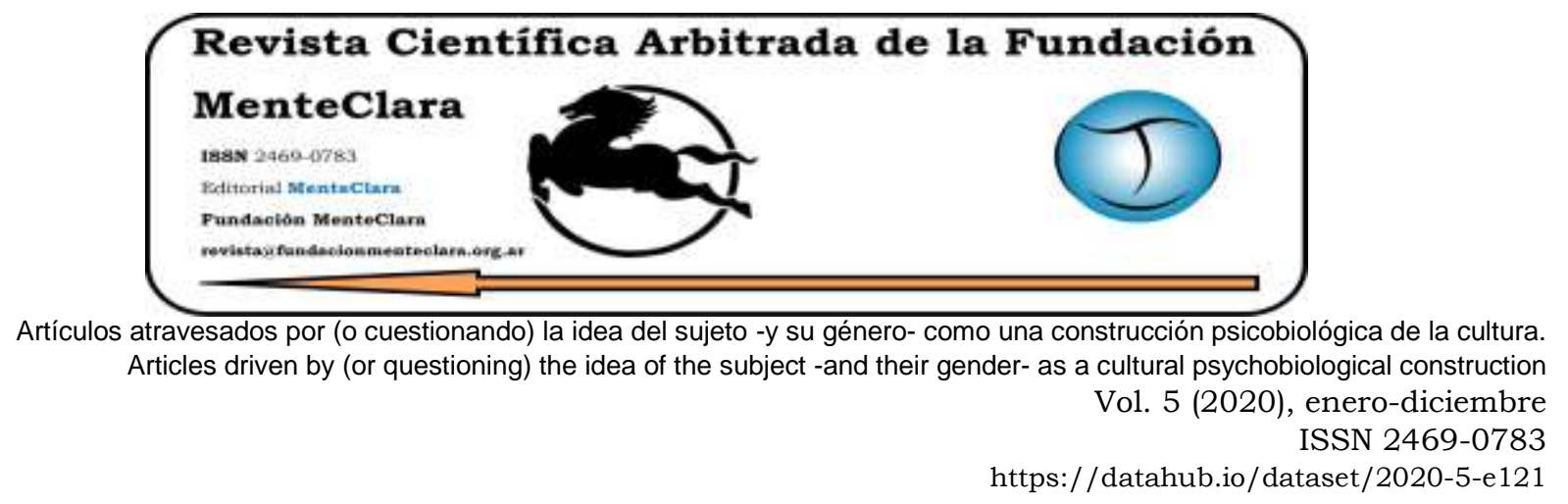

\title{
FAVALORO: EL INNOVADOR DETRÁS DE LA INNOVACIÓN
}

\author{
FAVALORO: THE INNOVATOR BEHIND INNOVATION
}

Leandro Clavero leandro@clavero.com.ar

Universidad Abierta Interamericana (UAI), Argentina

Cómo citar este artículo / Citation: Clavero, L. (2020). "Favaloro: El innovador detrás de la innovación". Revista Científica Arbitrada de la Fundación MenteClara, Vol. 5 (121). DOI: https://doi.org/10.32351/rca.v5.121

Copyright: (C) 2020 RCAFMC. Este artículo de acceso abierto es distribuido bajo los términos de la licencia Creative Commons Attribution 4.0 International License (CC BY 4.0). Recibido: 02/12/2019. Aceptado: 06/12/2019 Publicación online: 06/01/2020

Conflicto de intereses: Ninguno que declarar.

\section{Resumen}

Este artículo recorre la vida de uno de los grandes hombres del siglo XX. El Dr. René Favaloro, un gran hombre, a la luz de los sistemas de innovación. Se procedió para ello a una investigación documental en la cual se indagó en cada instancia de su vida y como se comportaron los diferentes elementos del sistema. El desarrollo de los procesos de aprendizaje y de la creación de capacidades que llevó al diseño e implementación de las innovaciones y la identificación de los principales interesados involucrados en dicho proceso de innovación. De esta manera se determinó que si los elementos políticos y financieros no acompañan, ni siquiera la presencia de un huracán innovador puede permitir que los logros alcanzados continúen más allá de su impulsor.

\section{Abstract}

This article traces the life of one of the greatest men of the 20th century. Dr. René Favaloro. The remarkable man in the threshold of the innovation systems. A documentary research was done in which it was inquired every instance of his life 
and how the different elements of the enviroment behaved. The development of the learning processes. The creation of skills that led to the design and the implementation of the innovations. The identification of the key stakeholders involved in this innovation process. In this way it was determined that if the political and financial factors do not accompany not even the presence of a hurricane innovative could allow the achievements to continue beyond its driver.

Palabras Claves: Innovación; Sistemas de Innovación; Favaloro

Keywords: Innovation; Innovation Systems; Favaloro 


\section{Introducción}

Si hablamos de innovación, es muy fácil pensar en el Dr. René Favaloro y en el Bypass. Pero detrás del creador de la cirugía cardiovascular existía un hombre comprometido con la innovación y el desarrollo.

Médico rural, ciudadano con compromiso social, docente, investigador, historiador. Excelente persona con enormes cualidades humanas pero que nunca supo ser ni político ni empresario.

Schumpeter definía innovación como "el desarrollo de un nuevo producto o proceso por parte de una empresa y su introducción exitosa en el mercados, el desarrollo de nuevas formas de organizar los negocios, nuevas fuentes de aprovisionamiento o la explotación de nuevos mercados" (Suárez 1, 2017). Si de esa concepción original extraemos la idea de negocios, Favaloro cumplió con todos los tipos de innovación.

"Se puede establecer que el sistema de innovación está conformado por -así como se retroalimenta- de: 1) El subsistema científico; 2) El subsistema de educación y formación; 3) El subsistema financiero y 4) las diferentes facetas de intervención estatal" (Anlló \& Suárez, 2008). A lo largo de la vida de este gran hombre, estos sub sistemas han funcionado de manera distinta permitiendo o limitando aquellas iniciativas que ha intentado impulsar.

A los fines de este trabajo, nos centraremos en 4 momentos de su vida que nos permitirán entender cabalmente quién fue y cómo fue su vínculo con la innovación: Su adolescencia y formación social, El médico rural, La cumbre de su carrera, El regreso y su muerte.

Desde sus inicios, el enfoque de los sistemas nacionales de innovación ha estado fuertemente preocupado por la identificación de criterios de política capaces de traccionar procesos de crecimiento y desarrollo (Suárez \& Erbes, 2014). Entendiendo ésto, recurriremos a este abordaje 
de la realidad para ver de qué forma actuaron para el desenlace de cada una de las etapas que recorreremos en el presente trabajo.

Favaloro fue lo que Shcumpeter, en Mark I, llamaria "El actor central del proceso de destrucción creativa, el emprendedor, quien veía en la ciencia el potencial de implementación de algún desarrollo y lo transformaba en una innovación" (Suárez 1, 2017). Así fue que construyó nuevas realidades por donde pasó.

\section{Su adolescencia y formación social}

Proveniente de una familia humilde y trabajadora, estudió en una escuela primaria pública y cursó su secundario en el Colegio Nacional de La Plata. En la misma ciudad también se formó como médico (Favaloro F., 2017).

"En la secundaria, sus docentes le infundieron principios sólidos de profunda base humanística. Más allá de los conocimientos que adquirió, incorporó y afianzó ideales como libertad, justicia, ética, respeto, búsqueda de la verdad y participación social, que habia que alcanzar con pasión, esfuerzo y sacrificio." (Favaloro F., Biografia - Los ideales que Guiaron sus Pasos)

En varias entrevistas sostuvo en forma clara lo que le habría inculcado Joaquín V. González en su adolescencia "No concibo a un estudiante universitario sin compromiso social" (Favaloro, 2010).

El platense estaba convencido de que la educación es el principal programa contra la desigualdad social y que la prosperidad de un país no se consigue en forma azarosa o individual, sino a través del trabajo y esfuerzo colectivo. (Manes, 2016). El conocimiento que se obtenía en las aulas de sus maestros debía compartirse y colaborar para que la sociedad pudiese crecer. Él entendía "el conocimiento como un elemento esencial en 
el proceso de desarrollo" (Anlló \& Suárez, 2008) y en su adultez escribió un libro titulado "Don Pedro y la Educación" en donde plasma su visión sobre ese tema y lo hace en honor a su profesor Pedro Enrique Sureña.

\section{E1 médico Rural}

Al finalizar sus estudios médicos, le ofrecieron un alto cargo universitario con la sola condición que dejara por escrito su adhesión politica con la ideología de las autoridades de la facultad de medicina. Sus principios lo llevaron a rechazar esa condición y emprendió un corto viaje que le demandó 12 años.

Se mudó a Jacinto Aráuz, un pequeño pueblo de La Pampa. Si toda mejora tecnológica $u$ organizacional que implica una novedad para la firma es una innovación (Suárez, 2008). Los Dres. Favaloro -René y su hermano- no dejaron de producir innovaciones durante toda su estadía allí.

Se introdujeron en un entorno muy carenciado, pocos recursos, poca infraestructura, poco dinero, muchas necesidades. "La búsqueda de mejoras en las rutinas, ya sea deliberadamente o como sub producto de la resolución de problemas, es lo que se denomina proceso de innovación" (Suárez 3, 2017). Fue así como buscando solucionar los problemas sanitarios y hacerse de recursos e infraestructura, el médico creo una clínica en donde todo aquel que lo necesitara sería atendido. Luego, re invirtiendo lo que allí ganaba logró introducir el primer equipo de rayos $\mathrm{x}$ de la región que lo importó directamente de Holanda. Este aparato es el mismo que hoy sigue funcionando allí.

Otro cambio radical para aquel poblado fue la creación de la Cooperativa de Obras y Servicios Públicos ya que su equipo de rayos $\mathrm{x}$ requería mayor potencia que la disponible para funcionar. Además de 
traer la electricidad, consiguieron traer agua potable. Estas mejoras permitieron erradicar una diarrea infantil que afectaba a toda la población.

Encontró en una iglesia de pueblo, el salón más grande de aquella zona. Alli comenzó a impartir charlas a todo el mundo sobre cómo cuidar la salud. "La innovación no puede pensarse disociada de los agentes ni éstos de su entorno" (Suárez, 2008). La innovación, el aprendizaje y el cambio tecnológico deben ser abordados desde una perspectiva sistémica (Barletta, Robert, \& Yoguel, 2014).Fue así que comenzó a transmitir sus saberes.

A su vez, tomó de aliados a los maestros rurales y a las comadronas. A estos actores les dedicó mucha energía a formar y colaborar. Puesto que el desarrollo implica la interacción de las habilidades sociales y el uso productivo del conocimiento (Natera, 2016). El avance técnico, como fuente de desarrollo, depende de la formación de recursos humanos y de la creación de un entorno sinérgico (Suárez, 2008) Fue así que esa red que armó, posibilitó bajar a cero la tasa de mortalidad infantil en ese pueblo alejado de La Pampa seca... (ElBiógrafo, 2017). Cuando el intendente de aquel entonces le preguntó cómo hizo para que los chiquitos no muriesen, él le contestó "sólo había que mezclarse con la gente y explicarle".

Según Lundvall el Sistema de Innovación es "un sistema social constituido por elementos y relaciones que interactúan para producir, difundir y usar conocimientos nuevos, económicamente útiles" (Erbes \& Suárez, 2016). Allá a 200km de la ciudad de Santa Rosa, se trabajó y fortaleció ese sistema de innovación circunscripto a unos pocos poblados

"La necesidad de coordinar agentes de diversas naturalezas $e$ intereses en una visión conjunta de desarrollo obliga a pensar en la 
incorporación del ámbito politico como herramienta de negociación y solución de conflictos: habrá que hacer uso del poder para aliviar las tensiones, configurar agendas compartidas, distribuir los beneficios $y$ daños" (Natera, 2016).

Es interesante observar cómo el trabajo de los Dres. Favaloro generó una revolución en el pueblo en el que trabajaron. Pero a pesar de ello, hoy el pueblo se encuentra en las mismas condiciones desde que los médicos se fueron. El desarrollo de las capacidades instaladas no pudo continuarse al no existir politicas públicas que no requirieran del empuje de ese transformador de realidades.

Aquí se observa que "la dependencia de la historia y la no reversibilidad se consideran fundamentales para explicar el proceso de innovación y desarrollo económico" (Natera, 2016). "La innovación como un medio para la mejora generalizada en el nivel de vida de la población" (Erbes \& Suárez 2, 2016), al no modificarse el entramado sistémico político en el cual estaba inserto ese pueblito, las mejoras generalizadas se estancaron.

\section{La cumbre de su carrera}

Favaloro vivió 10 años en Estados Unidos. Allí tuvo la posibilidad de colaborar, aprender, trabajar y enseñar con grandes mentes de la medicina. La innovación a nivel firma puede entenderse como la combinación de sus rutinas y capacidades sumadas a la selección del entorno. Las primeras son las formas estándar de hacer las cosas y las segundas son el agregado de los individuos junto con su trayectoria personal y colectiva. (Suárez 3, 2017). El médico argentino estudió todas las coronografias disponibles en la biblioteca del centro donde se formaba que era la colección más grande del mundo. Fue así cómo se introdujo 
en una organización con los mejores profesionales y grandes recursos obtenidos a lo largo del tiempo.

Según el Dr. Fernando Cichero, presidente del Colegio Argentino de Cirujanos Cardiovasculares, Favaloro creó la cirugía coronaria puesto que previo a su Bypass las técnicas eran muy rudimentarias. (Favaloro F., 2017).

Según Favaloro, menciona que en el año 1910 Alexis Carrel hacía Bypass en perros con una técnica rudimentaria que no lograba la sobrevivencia de los animales (Legrand, 1990). Según el Dr. René su contribución fue analizar los factores, regularizar la técnica, difundirla por el mundo (Legrand, 1990).

El doctor Alexis Carrel -premio Nobel de medicina en 1912-, es el padre de toda la cirugía arterial. Es interesante cómo surgió su labor que aprendió de una bordadora. En Francia le pegaron una puñalada al presidente Carnot, le cortaron una vena muy importante en el hígado, y Carrel, como estudiante, vio cómo ese presidente se moría y nadie sabía suturar esa vena que estaba rota. Por eso empezó sus trabajos experimentales de sutura de vasos. Hacía trasplantes de riñón, de bazo, de corazón, en animales a principios de este siglo, 1910 (Supervielle, 2017)

Aquí podemos observar la distinción schumpeteriana entre Invención, innovación y difusión. (López, 1996). A su vez, queda marcada la importancia de los procesos históricos y la acumulación creativa de un campo del saber para lograr la innovación a partir de los descubrimientos preliminares de otros.

"No es grato ser un novato frente a los nuevos equipos después de haber sido un experto en los antiguos" (Pérez, 1998) así lo demostraron los especialistas coronarios de aquel entonces. Desestimaron y criticaron el 
nuevo método hasta que una prestigiosa publicación médica sostuvo que el Bypass Coronario era el mejor método creado hasta el momento. Las fuerzas endógenas intentaron evitar el fortalecimiento de esta discontinuidad técnica, pero la calidad de la nueva técnica, el abanico de posibilidades que abria y los grandes médicos que auspiciaron de docentes le permitieron abrirse paso e instalar ese nuevo paradigma.

\section{El regreso y su muerte}

Favaloro sabía que una vez instalado el nuevo paradigma "serán la distribución del conocimiento, la calidad y la profundidad de la educación y de la adquisición de destrezas, la capacidad general para la innovación y la creatividad, las que determinarán las diferencias entre empresas y naciones, diferenciando las exitosas de las que quedarán atrás" (Pérez, 1998). Fue por ello que decidió volver a su país cuando estaba en lo más alto de la medicina mundial. Una vez en su tierra, trabajó incansablemente en su fundación en donde enseñó, investigó y operó hasta sus últimos días.

“El despliegue de una revolución tecnológica tiene efectos económicos y sociales tremendamente disparejos" (Pérez, 1998). De esta manera, en la Clínica Favaloro se decidió no preocuparse por si el paciente tenía los recursos para la intervención. Quien lo necesitara sería atendido, luego se analizaría la forma de pagar como era debido al personal interviniente y los gastos de la clínica. Su enorme conciencia social lo llevó a dejar los aspectos económicos en un segundo plano: primero la salud.

En los inicios del Bypass se utilizaron venas provenientes de los muslos. A modo explorativo se emplearon también venas de otras partes del cuerpo humano. Transcurridos 10 años se dieron cuenta que las cirugias realizadas con arterias de la zona toráxica funcionaban mejor 
que aquellas las efectuadas con las venas del muslo (Legrand, 1990). Se apuntó al seguimiento de los diferentes procesos, su medición y su aprendizaje. "La existencia de path dependence significa que el proceso de producción, distribución y competencia en el mercado envía información a la firma que retroalimenta sus decisiones y en función de ello en el periodo siguiente decide modificar o no su conducta" (Suárez 3, 2017). Luego de esa primera década de la Clínica Favaloro, los tejidos con los que se trabajaron fueron mutando. Este proceso también está vinculado con el Learning by doing (Suárez, 2008)

"Una revolución tecnológica no es simplemente un conjunto de nuevas técnicas... Se trata de una creciente constelación de innovaciones interdependientes en lo técnico, lo organizativo, lo gerencial y lo social" (Pérez, 1998). Es así como para poder llevar a cabo la innovación del Bypass aorto coronario, Favaloro debió crear nuevo instrumental quirúrgico. Estos elementos eran más precisos que los existentes hasta entonces y que habian sido concebidos para otro tipo de intervenciones. “El progreso técnico se define, generalmente en términos de la curva de posibilidades de producción que se mueve y/o en términos del creciente número de bienes posibles de ser producidos" (Dosi, 2003). El mundo entero comenzó a utilizar la innovación técnica y a emplear su instrumental. Pero su creador nunca patentó sus descubrimientos para que éstos puedan usarse libremente en beneficio de la sociedad. Grandes empresas comenzaron a producir en serie esa aparatología y a ganar cientos de millones de dólares. Esta renta extraordinaria nunca generó ganancias a su inventor.

En un momento, con 50 millones de dólares -pesos en ese momentopor cobrar, la Fundación se hizo inmanejable. Favaloro tuvo ofertas de coimas para obtener fondos públicos pero se negó. En otras oportunidades había pedido ayuda a De la Rúa, varias veces. En una 
carta le dice: "Estimado Fernando, te escribo estas lineas porque nuestra fundación está al borde de la quiebra. (...) Necesitamos alrededor de 6 millones de pesos. No tengo conexiones con el empresariado argentino," (ElBiógrafo, 2017).

"los círculos virtuosos son a menudo tan difícil de desenmarañar como los círculos viciosos" (Dosi, 2003). Esto nos lleva a pensar en dos premisas:

1) La crisis que desencadenó en la muerte de Favaloro se da al final de las políticas de los años 90 y cercana a la crisis del 2001 -contexto-. Los determinantes macroeconómicos tienen un peso significativo en la dirección de los esfuerzos de las firmas (Anlló \& Suárez, 2008). “En los periodos de recesión el gasto en $C$ y T no sólo cae sino que además pierde prioridad respecto de otros gastos públicos más urgentes. Al mismo tiempo, la falta de financiamiento atenta contra las capacidades adquiridas y se producen retrocesos en materia tecnológica que no necesariamente serán compensados por los avances que puedan producirse durante el siguiente periodo de crecimiento" (Anlló \& Suárez, 2008). "los criterios económicos que actúan como selectores definen cada vez con mayor precisión los senderos a seguir realmente entre un conjunto mucho más grande de senderos posibles" (Dosi, 2003). Ante la crisis social que se vivía en aquel entonces, la salud coronaria no era una prioridad.

2) Ese gran científico carecía de dotes políticas y empresariales persona-. "Mayores niveles de cohesión en la red se corresponden con mayores niveles de capacidades en las empresas" (Pereira, Barletta, \& Suárez, 2016). Nuestro protagonista supo estar en el centro de la vida mundial pero muy alejado de los círculos de poder político y económico. 
De esta manera, debiéramos pensar que el exterior y el interior de ese innovador condujeron a que él no pudiera continuar con su dantesca y solidaria contribución al desarrollo.

La interacción de la firma con su entorno es causa y consecuencia de las decisiones de las organizaciones (Suárez 4, 2017) y "Si la estrategia cambia, la estructura también cambiará y las capacidades deberán ser adaptadas" (Suárez 3, 2017). El mismo Favaloro se convirtió en la institución que no permitía la evolución de la organización. Para alcanzar un modo de gestión autosustentable decidió correrse, permitir que continúe su obra pero no participar de la solución. El 29 de julio de 2000 Fallece de un tiro en el pecho.

\section{Conclusión}

La capacidad médica y humana de René Favaloro no puede discutirse. Él nos permite entender en forma plena que la tecnología, cuando se emplea en forma correcta, lleva a un avance excepcional. (Supervielle, 2017)

A pesar de ello, nunca pudo entender que el contexto determina las vinculaciones, contiene las reglas de juego que se generan en un proceso no lineal (Natera, 2016). Él no aprendió a jugar el juego y el juego no supo aprovechar a ese gran jugador.

"El entorno se entiende como el conjunto de organizaciones públicas y privadas que impactan en el comportamiento innovador de las empresas y de los sistemas" (Suárez 4, 2017). Si el Estado hubiera colaborado en Jacinto Aráuz o si empresas privadas se hubieran acercado allí, su evolución seguramente hubiera continuado. Si el Estado no le hubiera debido a la Clínica Favaloro, si las empresas que ganaron fortunas con 
sus creaciones hubieran compartido una parte de sus ganancias. Otro sería nuestro cantar.

Dejamos pasar una ventana de oportunidad que se abrió ante nuestros ojos. Se creó la Cirugía Cardiovascular y su creador vino a la Argentina a colaborar con su país, enseñar e investigar. Pero las puertas las cerraron todas... menos la de atrás...

"Es necesario insistir una vez más que si no estamos dispuestos a comprometernos -principalmente los universitarios- a luchar por los cambios estructurales que nuestro pais y toda Latinoamérica demanda -principalmente en educación y salud- seguiremos siendo testigos de esta sociedad injusta donde parece que el tener y el poder son las aspiraciones máximas".

Dr. René Favaloro 


\section{Referencias}

Anlló, G., \& Suárez, D. (2008). Innovación: Algo más que I+D. Evidencias Iberoamericanas a partir de las encuenstras de innovación. Construyendo las estrategias empresarias competitivas. En AA.VV., El Estado de la Ciencia. Principales Indicadores de Ciencia y Tecnologías Iberoamericanas (págs. 73-103). RICyT, Buenos Aires.

Barletta, F., Robert, V., \& Yoguel, G. (2014). Tópicos de la teoría Evolucionista neoschumopeteriana de la innovación y del cambio tecnológico (Vol. 1). Buenos Aires, Argentina: Universidad Nacional General Sarmiento.

Dosi, G. (2003). Paradigmas y trayectorias tecnológicas. Una interpretación de las determinantes y direcciones del cambio tecnológico. (F. Chesnais, \& C. Neffa, Edits.) Ciencia, Tecnología y Crecimiento Económico, 99-198.

ElBiógrafo, H. (2017). Metro 951. Recuperado el 29 de 11 de 2019, de https://www.metro951.com/perros/rene-favaloro-by-harry-elbiografo/

Erbes, A., \& Suárez, D. (2016). Introducción. En A. Erbes, \& D. Suárez, Repensando el Desarrollo Latinoamericano. Una discusión de los sistemas de innovación (pág. 396). Buenos Aires, Argentina: Universidad Nacional General Sarmiento.

Erbes, A., \& Suárez (2), D. (2016). Nuevas preguntas para una nueva agenda de desarrollo. En A. Erbes, \& D. Suárez, Repensando el desarrollo Latinoamericano. Una discusión desde los sistemas de innovación (pág. 396). Buenos Aires, Argentina: Universidad Nacional General Sarmiento.

Favaloro, F. (s.f.). Fundación Favaloro. Recuperado el 8 de 12 de 2017, de https://www.fundacionfavaloro.org/biografia

Favaloro, R. (2010). YouTube. (J. elmenosloco, Ed.) Recuperado el 29 de 11 de 2017, de https://www.youtube.com/watch?v=SA4gaY8Ho3U

Legrand, M. (1990). YouTube. Recuperado el 2017 de 11 de 29, de https:/ / www.youtube.com/watch?v=pmk27yw_Vbc

López, A. F. (1996). Las ideas evolucionistas en economía: una visión en conjunto". Revista Buenos Aires Pensamiento Económico $\left(1^{\circ}\right), 28$.

Manes, F. (2016). Favaloro: la ciencia y el compromiso social. Recuperado el 29 de 11 de 2017, de https://www.cronista.com/infoespecial/Favaloro-la-ciencia-y-el-compromisosocial-20160707-0028.html 
Natera, J. M. (2016). Las dinámicas de los sistemas nacionales de innovación. En A. Erbes, \& D. Suárez, Repensando el Desarrollo Latinoamericano. Una Discusión desde los sistemas de Innovación (pág. 396). Buenos Aires, Argentina: Universidad Nacional General Sarmiento.

Pereira, M., Barletta, F., \& Suárez, D. (2016). El Rol de las vinculaciones en el desarrollo de las capacidades de la firma. Un análisis de las redes sociales de la industria del software en Argentina. Revista Pymes, Innovación y Desarrollo, 3(2-3), 3-21.

Pérez, C. (1998). El Reto Sociopolítico del cambio de paradigma tecnoeconómico. Revista del Banco Central de Venezuela, VIII, 12.

Suárez (1), D. (2017). El Concepto de Innovavión. Clase 1, FLACSO Virtual, Buenos Aires

Suárez (3), D. (2017). Innovación a Nivel Firma. Clase 3, FLACSO Virtual, Buenos Aires.

Suárez (4), D. (2017). El Enfoque sistémico de la innovación. Clase 4, FLACSO Virtual, Buenos Aires.

Suárez, D. (2008). Empresa, Innovación y competitividad: De la renta monopólica al desarrollo sustentable. Documento de Trabajo, Centro Redes, Buenos Aires.

Suárez, D., \& Erbes, A. (2014). Desarrollo y sub desarrollo latinoamericano. Un análisis crítico del enfoque de los sistemas de innovación para el desarrollo. Revista REDES, 20(38), 97-119.

Supervielle, O. B. (2017). René Favaloro: "Tengo miedo a que los jóvenes no tengan ilusiones ni sentido de un futuro mejor". La Nación, http:/ / www.lanacion.com.ar/2072688-rene-favaloro-tengo-miedo-a-que-los-jovenes-notengan-ilusiones-ni-sentido-de-un-futuro-mejor. 